\title{
The psychological and cognitive impact of Covid-19 on individuals with neurocognitive impairments: research topics and remote intervention proposals
}

\author{
Maria Devita ${ }^{1} \cdot$ Alessandra Bordignon $^{1} \cdot$ Giuseppe Sergi $^{1} \cdot$ Alessandra Coin $^{1}$
}

Received: 25 May 2020 / Accepted: 16 June 2020 / Published online: 24 June 2020

(c) Springer Nature Switzerland AG 2020

Keywords Covid-19 . Dementia $\cdot$ Quarantine $\cdot$ Psychological impact $\cdot$ Cognitive impact

\section{Introduction}

The novel coronavirus (Covid-19) that emerged in Wuhan, China, in December 2019 rapidly spread within Hubei province, and by January 30, 2020 had extended to up to 20 countries. Highly contagious and dangerous, Covid-19 quickly impacted governments and public health systems, so that almost the entire world had to adopt the clinical and social tools that had previously been successfully deployed on a massive and unprecedented scale in China. Quarantine, social distancing, and community containment are the public health measures that have recently, as in the past, been taken to separate people to interrupt transmission and thereby prevent person-to-person spread of this disease [1]. However, quarantine brings with it also important psychological sequelae, as recently reported in the general Chinese population [2], so that the World Health Organization (WHO) promptly provided guidelines for dealing with this problem. An increasing amount of data is becoming available on the psychological and social problems resulting from Covid-19 and the interventions to tackle them in the general population [3]; yet little attention is being paid to elderly population and, in particular, to a frail subgroup of this age: individuals with neurocognitive disorders. The "stayhome" disposition and the sudden upheaval of daily life routines are in themselves difficult to tolerate by healthy and/or younger people, but can become even more burdensome for older persons with diseases that involve cognitive impairments, no less. For them, a daily routine is fundamental as a behavioral

Maria Devita

maria.devita@unipd.it

1 Geriatrics Division, Department of Medicine, DIMED, University of Padua, Via Giustiniani 2, 35128 Padua, Italy therapeutic approach to control the typical Behavioral and Psychological Symptoms of Dementia (BPSD) of disease, as psychomotor agitation, wandering and aggression. The opportunity to leave the house and to go out is a great source of indirect cognitive stimulation, thanks to the variety of environmental stimuli and the multiple possible chances of social exchange. Finally, also the possible motor impairments associated to quarantine should not be underestimated: a forced reduction of motor/physical activity can cause, particularly in older individuals with neurocognitive disorders, a progressive loss of personal and instrumental autonomy, as well as a possible worsening of other agingrelated clinical problems, as sarcopenia, with a consequent increased risk of falls, and subsequent medical geriatrics complications.

With this background, the present Point of View paper aims to focus on a particularly frail population, namely on individuals with neurocognitive disorders, that have been relatively neglected up to now by the literature on the Covid19 pandemic. To do that, on a first section, this paper puts forward research topics aimed at deeper investigation of the indirect cognitive and psycho-affective effects of Covid-19 quarantine on individuals with neurocognitive disorders. Beyond providing selected points for discussion and highlighting some open issues, this paper suggests to consider remote assessment tools to be implemented not just during extraordinary periods, such as that of Covid-19, but also as a future and possible method of clinical practice. In the second section, "remote interventions" (RI) are proposed to be validated to provide neuropsychological assistance to persons with neurocognitive disorders, but also psychological and psychoeducational support for their caregivers during the pandemic but also for the next future. 


\section{Research topics}

\section{Remote assessment tools}

A first issue that we believe should be carefully considered results directly from the impositions of Covid-19 quarantine: the need of developing and/or standardizing remote assessment tools for older people with neurocognitive impariments. Although concerns may arise about the actual feasibility and reliability of such methodology, previous studies showed how diagnostic interviewing and several neuropsychological tests (e.g., the MMSE, verbal fluency, the Clock Test) conducted "via video teleconference and traditional face-to-face conditions have good agreements even with persons with cognitive impairments and dementia" [4]. During the lockdown, a number of clinicians and researchers have proposed research protocols, all motivated by the various scientific, clinical, social and psychological issues associated with Covid-19. However, so far, no one thought about remote neuropsychological evaluation, although it was proved to be previously successfully applied, clashing with the lack of measurements for collecting the necessary data instead, which poses a big challenge for the near future. The use of proxy reports, questionnaires administered by telephone (despite not being standardized for this application), and the creation of new "ad hoc" inventories could respond to the need for emergency tools able to overcome the social distancing in place to safeguard patient health. When Covid-19 quarantine ends, all the remote modalities deployed up till then will be evaluated and carefully considered; if found to be accurate, these new measures will likely constitute alternative, "at distance" methods that allow social distancing to be maintained; this is particularly important for older individuals. These new methods will also be of interest to all the Centers for Cognitive Decline and Dementia with large numbers of individuals to diagnose every day, and therapeutic prescriptions to renew periodically. Although the face-to-face clinical visit is undoubtedly always fundamental for cognitive impairment diagnosis and follow-up, remote assessment could be a valid transitional measure for public health needs in the coming months, and would guarantee continuity in patient care (against the currently imposed, though necessary, interruption), while reducing the risk of contagion and the consequent potential negative outcome of infection. The use of remote measurements could also be useful in speeding up and facilitating the massive collection of data that researchers and clinicians have to tackle daily.

\section{Open issues}

Older individuals are among the most frail populations exposed to Covid-19 contagion. As showed by the literature daily updated, it particularly concerns older people causing severe lungs and systemic inflammatory sequelae often requiring hospitalization and the subsequent, well known, related medical complications. This issue is even truer for individuals with neurocognitive disorders that are "the frailest among the frailest". For them, hospitalization, acute inflammatory status associated with possible marked hypoxia often triggers delirium, whose general negative impact is well known. Furthermore, even a milder Covid19 infection could cause collateral disturbances, such as hyporexia, certainly caused by the disease itself, but also due to the hypo-anosmia and dysgeusia often associated with this virus' infection and to a worsening of the nutritional status and malnutrition. Beyond these clinical and medical considerations, it should be highlighted that living with the Covid-19 pandemic could be really dramatic for these persons as they may have difficulties in remembering to wear a mask, or in understanding public health information and the constraints imposed, and hence in complying with them (it should be noted that some behavioral diseases, such as "wandering" or disinhibition, can aggravate the overall situation). These difficulties, aside from representing a further potential burden to caregivers, also place people with neurocognitive disorders to an increased risk of contagion, nourishing a dangerous vicious cycle.

A series of further questions seem in need of answering. For example, are individuals with cognitive impairment aware of the spread of Covid-19 and its effects? Are they able to experience distress as other people do? Could the potential lack of awareness (i.e., anosognosia) be a "protective" factor for people with cognitive impairments, so that, having lost part of their "world consciousness", they are not affected by fear, distress and psychological malaise? Or, on the contrary, could anosognosia be considered a major risk factor because it makes them less prone to the application of preventive measures and consequently they are more exposed to infectious risk with even serious complications? Is it possible that quarantine "forces" family members, like children and grandchildren, to give more constant care to older people? These changes can, of course, be a possible source of stress and behavioral problems. However, might it not also be that older people benefit from the greater proximity of their loved ones?

\section{Remote interventions}

Although great attention is paid to olders' biomedical care, fewer efforts are made for their psychological and cognitive well-being. This latter, in particular, should not be 
underestimated, since clinicians will potentially be witness of a population whose profile might be extremely changed during this period of pandemic. In particular, if Cognitive Stimulation Training (CST) is highly recommended for individuals with neurocognitive disorders, it merits even greater endorsement during Covid-19-related social isolation. Without dwelling on the well-known beneficial effects of CST on individuals with cognitive impairments, this Point of View paper would like to suggest that clinicians, and neuropsychologists in particular, to design and validate specific individual and group Remote CSTs. Given the impossibility of carrying out the routine clinical examinations that older people with cognitive impairment are involved in on a weekly basis, remote and online cognitive training sessions should be planned and carried out with the aim of maintaining general orientation-to-reality, and stimulating, albeit in a milder way, overall cognition. With the presence of a caregiver, an internet connection and the use of now widespread technological devices, CST could be carried out individually or in groups with ease and in comfort. The possibility of using this method in "normal" times is not excluded, as it would be a means of reaching those individuals who cannot physically take part in CST for organizational reasons (face-to-face interventions are undoubtedly to be preferred). According to previous studies using remote neuropsychological tools with people with neurocognitive disorders, specific technological expertise is not required by examiners. Neuropsychologists that already have the adequate conditions allowing in their clinical practice to carry out CSTs would substitute the "canonical" time for these face-to-face interventions with remote ones, thus not requiring extra resources in terms of time and costs. The Eurostat's statistics on information and communication technologies (ICTs) show that in 2016 close to half $(45 \%)$ of the elderly population-defined as those aged 65-74 years-in the EU-28 used the internet at least once a week. This figure could be compared with the situation a decade earlier in 2006, when just $10 \%$ of the elderly population was using the internet on a regular basis (at least once a week) [5]. It is true that a certain reticence still persists among older adults in using ICTs, specifically in Italy; however, data seem to suggest that this digital divide is being gradually overcoming.

\section{Information and education for carers of individuals with dementia}

Carers of individuals with dementia always deserve particular attention, but especially so in this extraordinary period of quarantine when caregiving in general-or cohabitationcan be extremely hard. With respect to "canonical" interventions in the care of people with cognitive impairments, successful, non-pharmacological treatment is desirable, as is actively involving caregivers in supportive psychological and "strategic" programs to deal with dementia. We propose making remote and online Information and Education sessions available to monitor patients' well-being and provide caregivers with, on the one hand, information and clarification regarding possible cognitive, behavioral and affective changes occurring during quarantine, and, on the other hand, the suggestions and strategies that clinicians normally give to their patients during routine practice, and that are probably needed even more in these times.

\section{Conclusions}

The extraordinary spread of Covid-19 has brought—and will soon bring more-new, alternative methods for use in research and clinical practice with the elderly population. We believe that these represent an opportunity to reinforce classical geriatric and neuropsychological methodologies and approaches in studying normal and pathological aging, not only during the Covid-19 pandemic but also in the near future, by providing remote tools able to reach those individuals who do not have the physical, social and/or affective means of obtaining adequate and constant care.

Author contributions Conceptualization: MD; writing—original draft preparation: $\mathrm{MD}, \mathrm{AB}$; writing - review and editing: $\mathrm{MD}, \mathrm{AB}, \mathrm{GS}, \mathrm{AC}$; supervision: GS, AC.

Funding This research received no specific Grant from any funding agency in the public, commercial or not-for-profit sectors, and to our knowledge.

\section{Compliance with ethical standards}

Conflict of interest There are no conflicts of interest associated with this publication.

Human and animal rights This study was carried out with respect to human and animal rights.

Informed consent For this type of study, formal consent is not required

\section{References}

1. Wilder-Smith A, Freedman DO (2020) Isolation, quarantine, social distancing and community containment: pivotal role for old-style public health measures in the novel coronavirus (2019nCoV) outbreak. J Travel Med 27:a020

2. Wang C, Pan R, Wan X et al (2020) Immediate psychological responses and associated factors during the initial stage of the 2019 coronavirus disease (COVID-19) epidemic among the general population in china. Int J Environ Res Public Health 17:1729

3. Orrù G, Ciacchini R, Gemignani A et al (2020) Psychological intervention measures during the COVID-19 pandemic. Clin Neuropsychiatry 17:76-79 
4. Cullum CM, Hynan LS, Grosch M et al (2014) Teleneuropsychology: evidence for video teleconference-based neuropsychological assessment. J Int Neuropsychol Soc 20:1028-1033

5. Eurostat (2019) People in the EU- statistics on an ageing society. https://ec.europa.eu/eurostat/statisticsexplained/index.php/ People_in_the_EU_statistics_on_an_ageing_society\#Senior_citiz ens_online_.E2.80.94_silver_surfers. Accessed 14 Sep 2017
Publisher's Note Springer Nature remains neutral with regard to jurisdictional claims in published maps and institutional affiliations. 